\title{
MicroRNA-10b-5p downregulation inhibits the invasion of glioma cells via modulating homeobox B3 expression
}

\author{
WEILING LI, CHAOYING LI, QI XIONG, XIANG TIAN and QIN RU \\ Wuhan Institutes of Biomedical Sciences, Jianghan University, Wuhan, Hubei 430056, P.R. China
}

Received June 20, 2018; Accepted March 22, 2019

DOI: $10.3892 / \mathrm{etm} .2019 .7506$

\begin{abstract}
MicroRNA-10b (miR-10b) has been reported to be specifically upregulated in glioma tissues and cell lines. The aim of the present study was to investigate the effect of miR-10b-5p on the proliferation and invasion of glioma cells, and the possible underlying molecular mechanism. Cell viability was evaluated using an MTT assay, and flow cytometry was performed for cell cycle analysis. The effects of miR-10b-5p on glioma cell migration and invasion were assessed using wound healing and Transwell assays, respectively. The activity of matrix metalloproteinase 2 (MMP2) was also determined using zymography. Furthermore, homeobox B3 (HOXB3) mRNA expression in glioma cells was examined using quantitative polymerase chain reaction analysis. The protein expression levels of HOXB3, high mobility group box 1 (HMGB1) and Ras homolog family member C (RhoC) were further measured using western blotting. It was observed that glioma cells transfected with miR-10b-5p inhibitor exhibited significantly decreased proliferation. The wound healing and Transwell assays demonstrated that the miR-10b-5p inhibitor reduced the ability of glioma cells to migrate and invade, while transfection with miR-10b-5p mimic exhibited the opposite effect. HOXB3 was downregulated by miR-10b-5p at both the mRNA and protein levels. In addition, the expression of proteins associated with migration and invasion, including HMGB1, RhoC and MMP2, was upregulated in glioma cells transfected with miR-10b-5p mimic, while these proteins were downregulated in cells transfected with miR-10b-5p inhibitor. Taken together, the findings of the present study indicated that miR-10b-5p downregulation suppressed glioma cell proliferation and invasion, possibly by modulating HOXB3, which may provide a novel bio-target for glioma therapy.
\end{abstract}

Correspondence to: Dr Qin Ru, Wuhan Institutes of Biomedical Sciences, Jianghan University, 8 Sanjiaohu Road, Wuhan Economic and Technological Development Zone, Wuhan, Hubei 430056, P.R. China

E-mail: ruq.whibs@aliyun.com

Key words: microRNA-10b-5p, glioma, homeobox B3, proliferation, invasion

\section{Introduction}

Malignant glioma is the most common intracranial brain tumor, characterized by continuous growth, aggressive behavior, invasiveness and high genetic heterogeneity. The highly infiltrative and migrating behavior of glioma leads to diffuse growth and frequent recurrence of the tumor (1). The mean survival of patients with glioma is $\sim 14$ months after diagnosis, despite aggressive treatment with neurosurgery, radiotherapy and chemotherapy (2). Therefore, better therapeutic strategies, such as new molecular targets, are urgently required to effectively prevent glioma cell invasion.

Recent research revealed the important roles of microRNA-10b (miR-10b) processing in glioma cell growth, migration and invasion (3). miR-10b belongs to the miR-10 family and is located on chromosome $2 \mathrm{q} 31.1$, in the middle of the homeobox D (HOXD) cluster and upstream of HOXD4 (4). miR-10b has been reported to be highly and specifically expressed in glioblastoma tissues and glioma cell lines $(5,6)$. High expression of miR-10b has been associated with high-grade glioma and poor prognosis (7), whereas miR-10b downregulation suppressed the growth of subcutaneous and intracranial glioblastoma xenografts (8). In glioma, miR-10b downregulated several key cell cycle inhibitors and proapoptotic genes, such as Bim, TFAP2C, p16 and p21, indicating its function in promoting the cell cycle (9). In addition, miR-10b has been demonstrated to suppress TP53, PAX6, NOTCH1 and HOXD10 gene expression, which may coordinately regulate cancer invasion (10). Our previous study reported that miR-10b directly targeted Apaf-1 to regulate glioma cell apoptosis (11). Although several targets of miR-10b have been identified in glioma, the exact mechanism underlying the induction of glioma cell invasion by miR-10b remains unclear. Thus, the current study attempted to follow up on the role of miR-10b-5p in glioma, exploring its expression and functions on cell proliferation and invasion.

Based on sequence alignment with the miRBase database (www.mirbase.org/), miR-10b may target human HOXB3. HOXB3 is a transcription factor located on chromosome 17, which acts as a metastasis suppressor gene, and participates in cell apoptosis, proliferation, migration, invasion and epithelial-to-mesenchymal transition (12). Chen et al (13) reported that miR-10b targeted HOXB3 in endometrial cancer, which inhibited apoptosis and promoted cell proliferation, migration and invasion. Yang et al (14) also found 
that the upregulation of HOXB3 inhibited pancreatic cancer cell proliferation, migration and chemosensitivity. Therefore, it may be hypothesized that HOXB3 plays a functional role in glioma cells.

In the present study, to explore the effect of miR-10b on glioma cell proliferation and invasion, miR-10b-5p mimic or inhibitor was transfected into U87 and U251 cells. Reverse transcription-quantitative polymerase chain reaction (RT-qPCR) analysis was applied to assess the mRNA expression level of HOXB3 in glioma cells. Furthermore, the effect of miR-10b-5p on the expression of invasion-associated proteins in glioma cells was investigated using various methods, including western blotting and zymography. The results of the present study may provide an insight into the molecular mechanisms underlying the effect of miR-10b-5p in glioma cells.

\section{Materials and methods}

Cell lines and cell culture. The human glioma cell lines U251 (cat. no. TCHu 58) and U87-MG (glioblastoma of human origin, cat. no. TCHu138; Chinese Academy of sciences) were obtained from the National Infrastructure of Cell Line Resource (Shanghai, China) and grown in Dulbecco's modified Eagle's medium and RPMI-1640 medium (Gibco; Thermo Fisher Scientific, Inc., Waltham, MA, USA), respectively. Complete medium was supplemented with $10 \%$ fetal bovine serum (FBS), $100 \mathrm{U} / \mathrm{ml}$ penicillin and $100 \mathrm{mg} / \mathrm{ml}$ streptomycin (Gibco; Thermo Fisher Scientific, Inc.). The cell lines were cultured in an incubator at $37^{\circ} \mathrm{C}$ with a $5 \% \mathrm{CO}_{2}$ atmosphere. The cell lines were identified by the Genetic Testing Biotechnology Corporation (Suzhou, China) and represented a 94\% match with the cell lines of the DSMZ Reference Database.

Transient transfection. miR-10b-5p mimic, inhibitor and corresponding negative control were chemically synthesized by RiboBio Co., Ltd. (Guangzhou, China). The mimic and inhibitor sequences were as follows: miR-10b-5p mimic, 5'-UACCCUGUAGAACCGAAUUUGUG-3'; and control mimic, 5'-UUUGUACUACACAAAAGUACUG-3'; miR-10b-5p inhibitor, 5'-UACCCUGUAGAACCGAAUUUG UG-3', and control inhibitor, 5'-UCACAACCUCCUAGA AAGAGUAGA-3'. Prior to transfection, cells were plated at $70-80 \%$ confluence, and then transfection of oligonucleotides was performed using the Lipofectamine ${ }^{\mathrm{TM}} 2000$ reagent (Invitrogen; Thermo Fisher Scientific, Inc.). A total of $100 \mathrm{nM}$ mimic, $200 \mathrm{nM}$ inhibitor or corresponding control miRNA was added to each well. The cells were incubated for $48 \mathrm{~h}$ after transfection and then subjected to various assays.

Cell viability assay. U87 and U251 cells $\left(5 \times 10^{3}\right.$ cells/well) were seeded in triplicate into 96-well plates in $100 \mu \mathrm{l}$ complete medium. Cells were then transfected with miR-10b-5p mimic, miR-10b-5p inhibitor or control miRNA. After $48 \mathrm{~h}$ of incubation, cell viability was evaluated using an MTT assay. Approximately $20 \mu 1$ of $5 \mathrm{mg} / \mathrm{ml}$ MTT solution (Thermo Fisher Scientific, Inc.) was added to each well, and the samples were incubated for $4 \mathrm{~h}$ at $37^{\circ} \mathrm{C}$. Subsequently, the supernatant was carefully removed, and $150 \mu \mathrm{l}$ DMSO was added to dissolve the cells. The optical density at $570 \mathrm{~nm}$ was measured using a microplate reader (Thermo Fisher Scientific, Inc.).

Cell cycle analysis. U87 and U251 cells ( $1 \times 10^{5}$ cells/well) were seeded into 24-well plates and allowed to grow for $48 \mathrm{~h}$, followed by transfection with miR-10b-5p mimic or inhibitor. Cells were harvested by trypsinization, and then cell pellets were collected, washed twice with phosphate-buffered saline (PBS) and fixed with $70 \%$ ethanol for $3 \mathrm{~h}$ at $-20^{\circ} \mathrm{C}$. The fixed cells were washed once with PBS, mixed with an equal volume of Muse cell cycle reagent (Merck KGaA, Darmstadt, Germany) and then incubated for $30 \mathrm{~min}$ at room temperature in the dark. Then cells were analyzed by flow cytometry. The distribution of cells at each cell cycle phase was determined by Muse ${ }^{\circledR}$ Cell Analyzer using the Muse ${ }^{\circledR}$ Cell Cycle Assay kit (Merck KGaA), analyzing a mean number of 10,000 cells.

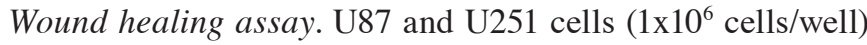
were seeded into 6-well plates. After reaching $70 \%$ confluence, the cells were transfected with miR-10b-5p mimic or inhibitor, and incubated for $48 \mathrm{~h}$. Using a $200-\mu 1$ sterile pipette tip, three scratches were created in each well (15). Next, the cells were washed twice with PBS and incubated in medium supplemented with $2 \%$ FBS for $24 \mathrm{~h}$. In order to assess the cell migration, images of the wells were captured at 0 and $24 \mathrm{~h}$ in three randomly selected microscopic fields (Olympus Corporation, Tokyo, Japan). The distance over which the cells had migrated into the denuded area was determined by measuring the wound width at 0 and $24 \mathrm{~h}$ using Image J software (National Institutes of Health, Bethesda, MD, USA) to calculate the healing rate.

In vitro migration and invasion assays. Cell migration and invasion were assayed using $8-\mu \mathrm{m}$ pore polycarbonate membrane Transwell chambers (Corning Costar, Corning, NY, USA). Briefly, U87 and U251 cells seeded in 6-well plates $\left(1 \times 10^{5}\right.$ cells/well) transfected with miR-10b-5p mimic or inhibitor for $48 \mathrm{~h}$ were transferred onto the upper Transwell chamber that was filled with $200 \mu \mathrm{l}$ serum-free medium for $24 \mathrm{~h}$. Complete medium $(600 \mu \mathrm{l})$ was added to the lower chamber of each well. For the Transwell invasion assay, the plate inserts used were precoated with Matrigel (BD Biosciences, San Jose, CA, USA). After $24 \mathrm{~h}$, the cells were washed three times with PBS, and non-migratory or non-invasive cells in the upper side of the chambers were removed with cotton swabs. The cells that had migrated or invaded were fixed in $4 \%$ paraformaldehyde and then stained with $0.5 \%$ crystal violet solution for $30 \mathrm{~min}$. Following washing twice with PBS, images of the cells were obtained at a magnification of $x 200$. Finally, the chambers were eluted by $33 \%$ acetic acid, and the optical density at $570 \mathrm{~nm}$ was measured.

Gelatin zymography. The activity of matrix metalloproteinase 2 (MMP2) in the miR-10b-5p mimic or inhibitor groups was analyzed in order to assess changes in the invasive ability of glioma cells. Briefly, supernatant fractions of cells cultured in serum-free medium for $24 \mathrm{~h}$ were concentrated using $10 \mathrm{kDa}$ Vivaspin 500 (Sartorius AG, Göttingen, Germany). Equal amounts of proteins were separated via 8\% SDS-PAGE with $0.1 \%$ gelatin under non-reducing conditions. Following the 
completion of electrophoresis, the gels were washed twice with renaturing buffer (containing $50 \mathrm{mM}$ Tris- $\mathrm{HCl}, \mathrm{pH} 7.6,5 \mathrm{mM}$ $\mathrm{CaCl}_{2}, 1 \mu \mathrm{M} \mathrm{ZnCl}_{2}$ and $2.5 \%$ Triton $\mathrm{X}-100$ ) within $30 \mathrm{~min}$ at room temperature. The gels were subsequently incubated in developing buffer (containing $50 \mathrm{mM}$ Tris- $\mathrm{HCl}, \mathrm{pH}$ 7.6, $5 \mathrm{mM}$ $\mathrm{CaCl}_{2}, 1 \mu \mathrm{M} \mathrm{ZnCl}_{2}, 200 \mathrm{mM} \mathrm{NaCl}$ and $0.02 \%$ Brij-35) at $37^{\circ} \mathrm{C}$ for $24 \mathrm{~h}$, washed in water, stained with Coomassie Brilliant Blue R-250 for $1 \mathrm{~h}$, and then destained with $40 \%$ methanol and $5 \%$ acetic acid until the bands were visible. Band intensities were quantitatively determined by ImageJ software.

miR-10b-5p target gene identification. Using bioinformatics software (TargetScan7.1; www.targetscan.org/vert_71/; and miRanda database; www.mirbase.org/), gene binding sites between miR-10b-5p and HOXB3 were detected.

$R T-q P C R$ analysis. Total RNA was isolated from the cultured cells using TRIzol reagent (Invitrogen; Thermo Fisher Scientific, Inc.). To quantify the relative content of miR-10b-5p and HOXB3 mRNA, $\sim \mu \mathrm{g}$ of RNA from each sample was reverse-transcribed using the Advantage RT-for-PCR kit (Takara Biotechnology Co., Ltd., Dalian, China). RT was conducted with oligo d(T)18 primers (Takara Biotechnology Co., Ltd.) and the miR-10b-5p RT primer, 5'-CTCAACTGGTGTCGTGGAGTC GGCAATTCAGTTGAGCACAAATT-3' (Genscript Biotech, Piscataway, NJ, USA). Next, qPCR was run using SYBR Green PCR Master Mix (Invitrogen; Thermo Fisher Scientific, Inc.) and specific primers for miR-10b-5p, U6, HOXB3 and $\beta$-actin on an iQ5 Sequence Detector (Bio-Rad Laboratories, Inc. Hercules, CA, USA). The primer sequences were as follows: miR-10b-5p, 5'-GGGTACCCTGTAGAACCG-3' (forward) and 5'-AACTGGTGTCGTGGAGTCGGC-3' (reverse); U6, 5'-CTC GCTTCGGCAGCACA-3' (forward) and 5'-AACGCTTCA CGAATTTGCGT-3' (reverse); HOXB3, 5'-ACCTTTCCCATC ACCCTT-3' (forward) and 5'-CGCTTCTTGGATTCTACC-3' (reverse); $\beta$-actin, 5'-ACACTGTGCCCATCTACG-3' (forward) and 5'-TGTCACGCACGATTTCC-3' (reverse). Subsequently, the comparative $2^{-\Delta \Delta \mathrm{Cq}}$ method (16) was used to quantify the gene expression levels. The relative expression of miR-10b-5p and HOXB3 mRNA was normalized to that of U6 and $\beta$-actin, respectively.

Western blotting. Radioimmunoprecipitation assay lysis buffer (Boster Biological Technology, Ltd., Wuhan, China) with freshly added protease inhibitor cocktail and phenylmethylsulfonyl fluoride was used to isolate the total protein from the cells. The protein concentration was then quantified using a BCA Protein Assay kit (Boster Biological Technology, Ltd.). Next, $30 \mu \mathrm{g}$ protein was loaded onto $10 \%$ SDS-PAGE and transferred onto polyvinylidene difluoride membranes (Merck Millipore, Billerica, MA, USA). Following blocking with $5 \%(\mathrm{w} / \mathrm{v})$ milk in tris-buffered saline containing $0.1 \%$ (v/v) Tween 20 (TBST) for $1 \mathrm{~h}$ at room temperature, the blots were probed with the following primary antibodies overnight at $4^{\circ} \mathrm{C}$ : HOXB3 (cat. no. abs139097-100 $\mu \mathrm{g}$; Absin Bioscience, Inc., Shanghai, China; dilution, 1:500), Ras homolog family member C (RhoC; cat. no. 3430S; Cell Signaling Technology Inc., Danvers, MA, USA; dilution, 1:1,000), high mobility group box 1 (HMGB1; cat. no. ab92310-100 $\mu \mathrm{l}$; Abcam, Cambridge, MA, USA; dilution, 1:1,000) and $\beta$-actin (cat. no. 4970S Cell
Signaling Technology, Inc.; dilution, 1:1,000). Subsequently, the samples were incubated with the appropriate secondary antibodies (horseradish peroxidase-conjugated anti-rabbit or anti-mouse IgG; cat. no. BA1054 and BA1050; Boster Biological Technology, Ltd., China; 1:3,000) for $1 \mathrm{~h}$ at room temperature. The bands were visualized with ECL substrate (Thermo Fisher Scientific, Inc.) using the Syngene G:BOX system (Gene Company, Ltd., Hong Kong, China). Each band was quantified by densitometry using Gen Tools software program (Gene Company, Ltd.), with $\beta$-actin used for normalization.

Statistical analysis. Statistical analysis for all the experiments was performed using Student's two-tailed t-test for comparison of two independent groups (experimental group and corresponding control group) with GraphPad Prism 5 software (GraphPad Software, Inc., La Jolla, CA, USA). Data from three independent experiments are presented as the mean \pm standard error of the mean. A P-value of $<0.05$ was considered to denote a statistically significant difference.

\section{Results}

miR-10b-5p downregulation inhibits the proliferation of glioma cells. As reported previously, miR-10b is significantly overexpressed in glioma cell lines (6). In the present study, RT-qPCR analysis was performed to examining the efficiency of mimic/inhibitor transfection and cell viability in glioma cells (Fig. 1A). Compared with the corresponding control group, the expression of miR-10b-5p was significantly increased in the mimic group, while that of the inhibitor group was decreased. A cell viability assay was also performed to investigate the effect of miR-10b-5p expression on the proliferation of glioma cells. In the miR-10b-5p inhibitor group, the cell viability was significantly lower at $48 \mathrm{~h}$ after transfection, reduced to $76.2 \pm 4.7$ and $80.2 \pm 7.1 \%$ of the control inhibitor group in U251 and U87 cells, respectively $(\mathrm{P}<0.05$; Fig. $1 \mathrm{~B})$. By contrast, no statistically significant difference in cell viability was detected between the miR-10b-5p mimic and control mimic groups.

To further explore how miR-10b-5p affects the growth of glioma cells, the cell cycle distribution of glioma cells transfected with miR-10b-5p mimic or inhibitor was analyzed. The data shown in Fig. 1C and D demonstrated that the fraction of U251 cells in G2/M phase was significantly decreased following inhibitor transfection $(7.9 \pm 0.5 \%)$ as compared with that in the control group $(12.7 \pm 1.3 \%$; $\mathrm{P}<0.05)$. By contrast, U87 cells accumulated in the $G_{0} / G_{1}$ phase of the cell cycle following transfection with miR-10b-5p inhibitor (70.9 $\pm 1.4 \%)$,

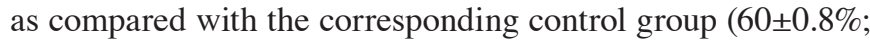
$\mathrm{P}<0.05)$. These data clearly indicated that miR-10b-5p downregulation affects cell cycle progression mainly by prolonging the $\mathrm{G} 2 / \mathrm{M}$ or $\mathrm{G}_{0} / \mathrm{G}_{1}$ phase. By contrast, transfection with miR-10b-5p mimic exerted no marked effect on the cell cycle distribution (Fig. 1C and D).

miR-10b-5p upregulation promotes glioma cell migration and invasion. The role of miR-10b-5p on glioma cell migration was further investigated. Glioma cells transfected with the miR-10b-5p mimic or inhibitor were used in the wound healing assay. The results indicated that miR-10b-5p inhibition 
A

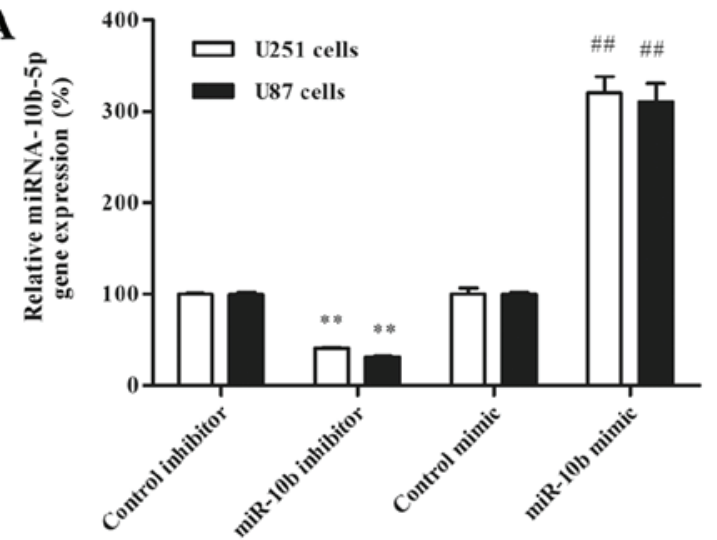

$\mathbf{C}$

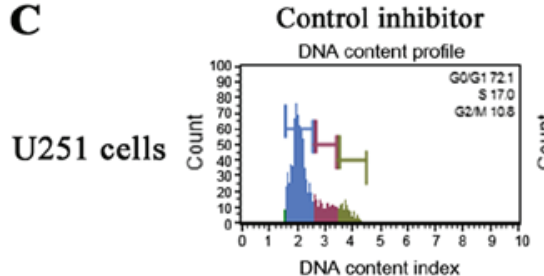

DNA content index
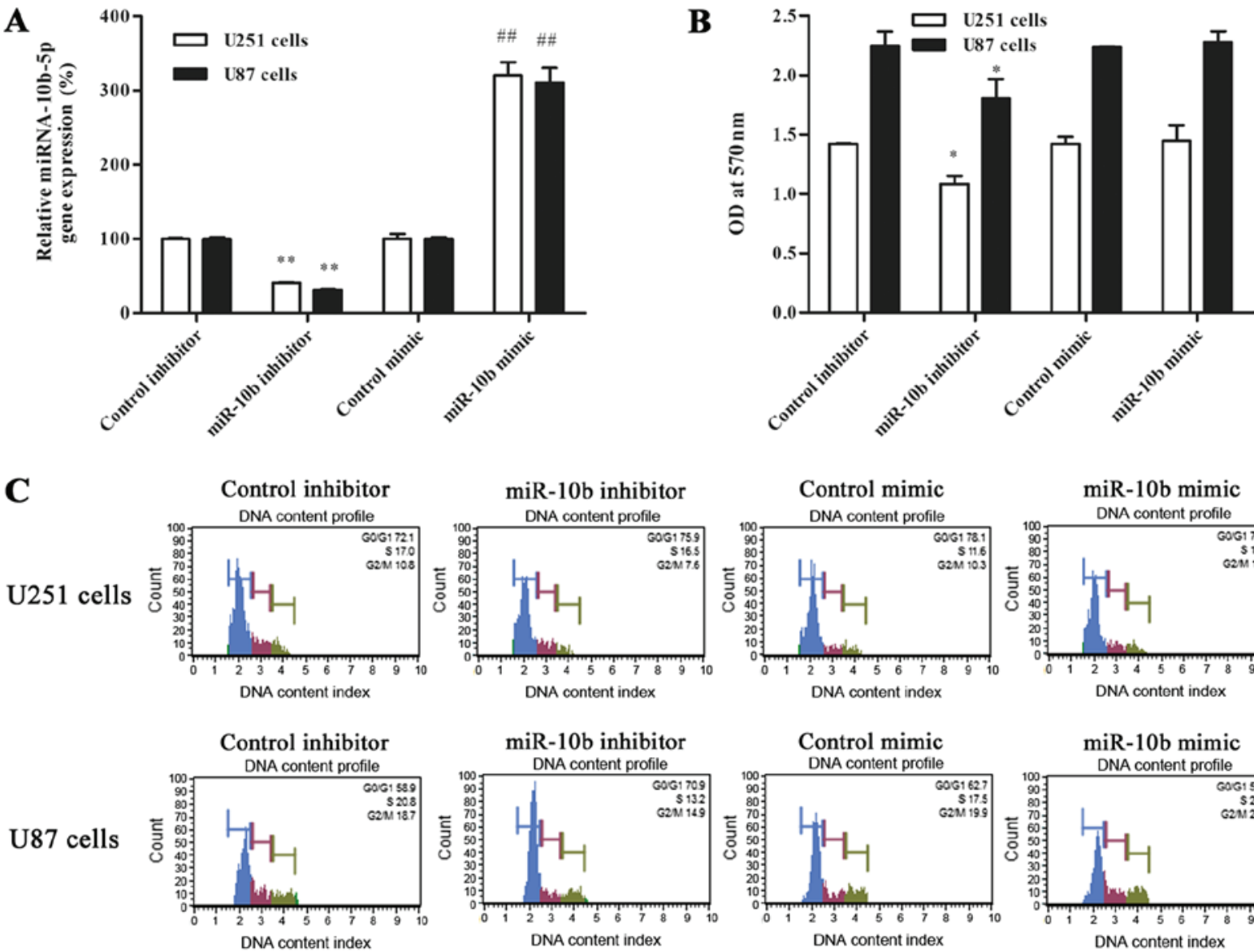

D

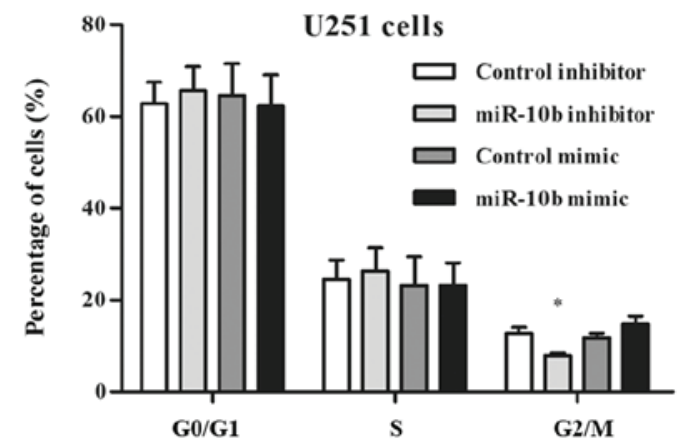

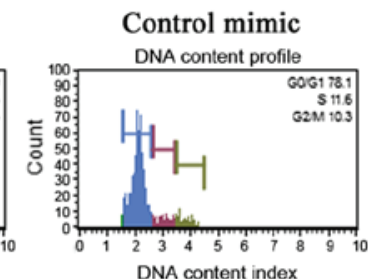

DNA content index
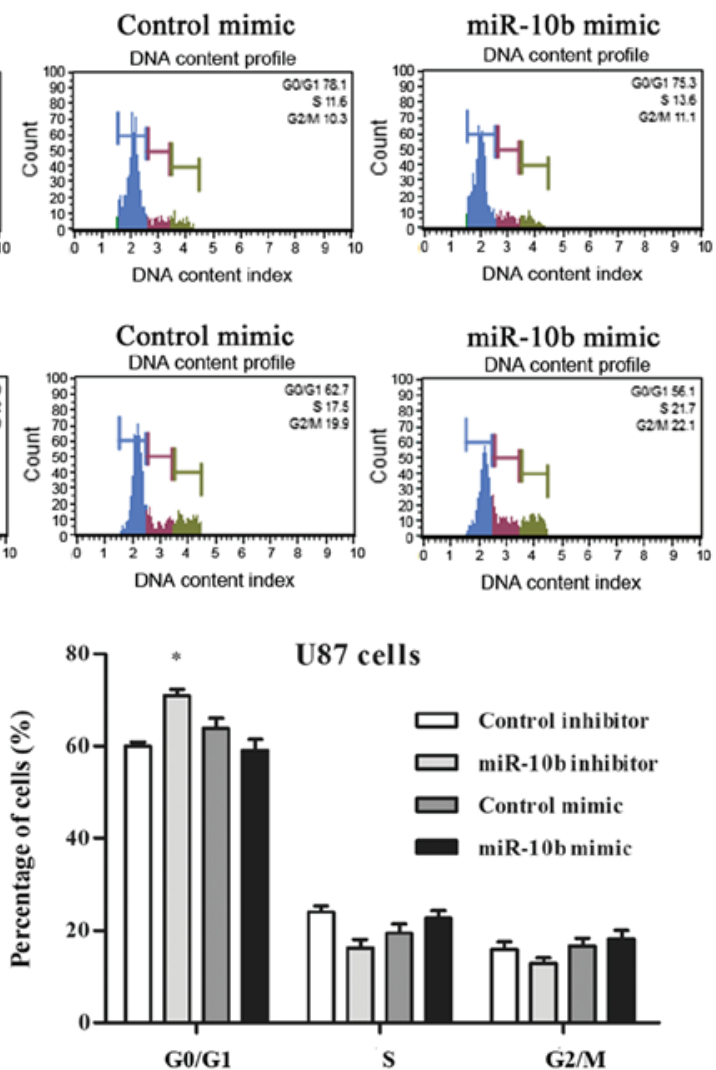

Figure 1. Effect of miR-10b-5p on glioma cell viability and cell cycle. (A) miR-10b-5p was subjected to reverse transcription-quantitative polymerase chain reaction analysis to detect miR-10b-5p and U6 expression levels. (B) Cell viability was suppressed following transfection with miR-10b-5p inhibitor in U251 and U87 cells. (C) Cell cycle analysis and (D) cell proportions (\%) in each phase of the cell cycle were determined in glioma cells transfected with miR-10b-5p mimic or inhibitor. miR-10b-5p inhibition induced cell cycle arrest at the $\mathrm{G}_{0} / \mathrm{G}_{1}$ or $\mathrm{G} 2 / \mathrm{M}$ phase. Data were analyzed by Student's $t-t e s t$. ${ }^{*} \mathrm{P}<0.05$ and ${ }^{* *} \mathrm{P}<0.01$ vs. control inhibitor group; ${ }^{\# \#} \mathrm{P}<0.01$, vs. control mimic group. miR, microRNA.

markedly suppressed the migration ability of U251 and U87 cells (Fig. 2A and B), with a reduction of $22.1 \pm 4.7 \%$ observed in U251 cells and $26.2 \pm 6.7 \%$ in U87 cells compared with the control inhibitor $(\mathrm{P}<0.05)$. By contrast, the migration ability in the miR-10b-5p mimic-transfected group was not evidently affected.

In addition, Transwell assay was performed to test the effect of miR-10b-5p on cell migration and invasion. The experiments demonstrated that the number of glioma cells migrating through non-coated and Matrigel-coated Transwell membranes was significantly reduced following inhibition of miR-10b-5p expression. As presented in Fig. 3A-C, a $41.1 \pm 5.6 \%$ reduction in the migration of $\mathrm{U} 251$ cells and a $39.4 \pm 3.4 \%$ reduction in U87 cells were observed compared with the control inhibitor group $(\mathrm{P}<0.01)$. Similarly, miR-10b-5p inhibitor led to a $30.3 \pm 3.3 \%$ reduction in invasion in $\mathrm{U} 251$ cells and a $44.2 \pm 2.0 \%$ reduction in U87 cells, as compared with the control inhibitor group $(\mathrm{P}<0.01)$. By contrast, miR-10b-5p mimic transfection significantly increased the migration and invasion of glioma cells in comparison with those in the control mimic group.

Overexpression of MMP2 and its extracellular activation, as well as its pro-invasion function, have been widely reported in glioma (17). To investigate the effect of miR-10b-5p on the gelatinase activity of MMP2 in glioma cells, gelatin zymography was performed. As shown by zymography analysis (Fig. 4A and B), MMP2 secretion was significantly reduced in the miR-10b-5p 

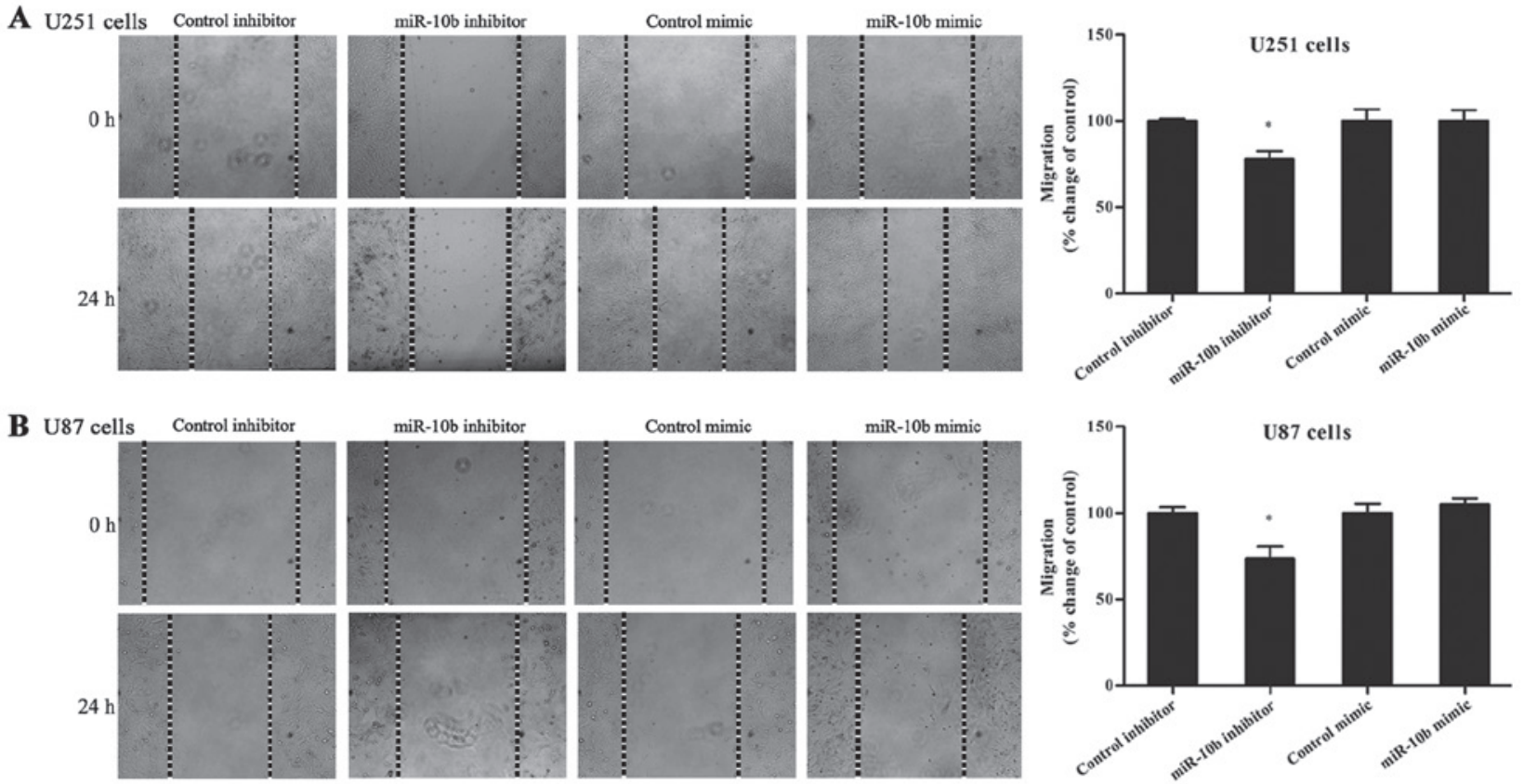

Figure 2. miR-10b-5p regulates glioma cell migration. (A) U251 and (B) U87 cell migration was significantly reduced in the miR-10b-5p inhibitor group. The left panel displays images of the wound healing assay (magnification x100), while the right panel presents a graphic presentation of the mean healing rates. Relative migration was quantified and compared with the corresponding control group. " $\mathrm{P}<0.05$ vs. control inhibitor group. miR, microRNA.
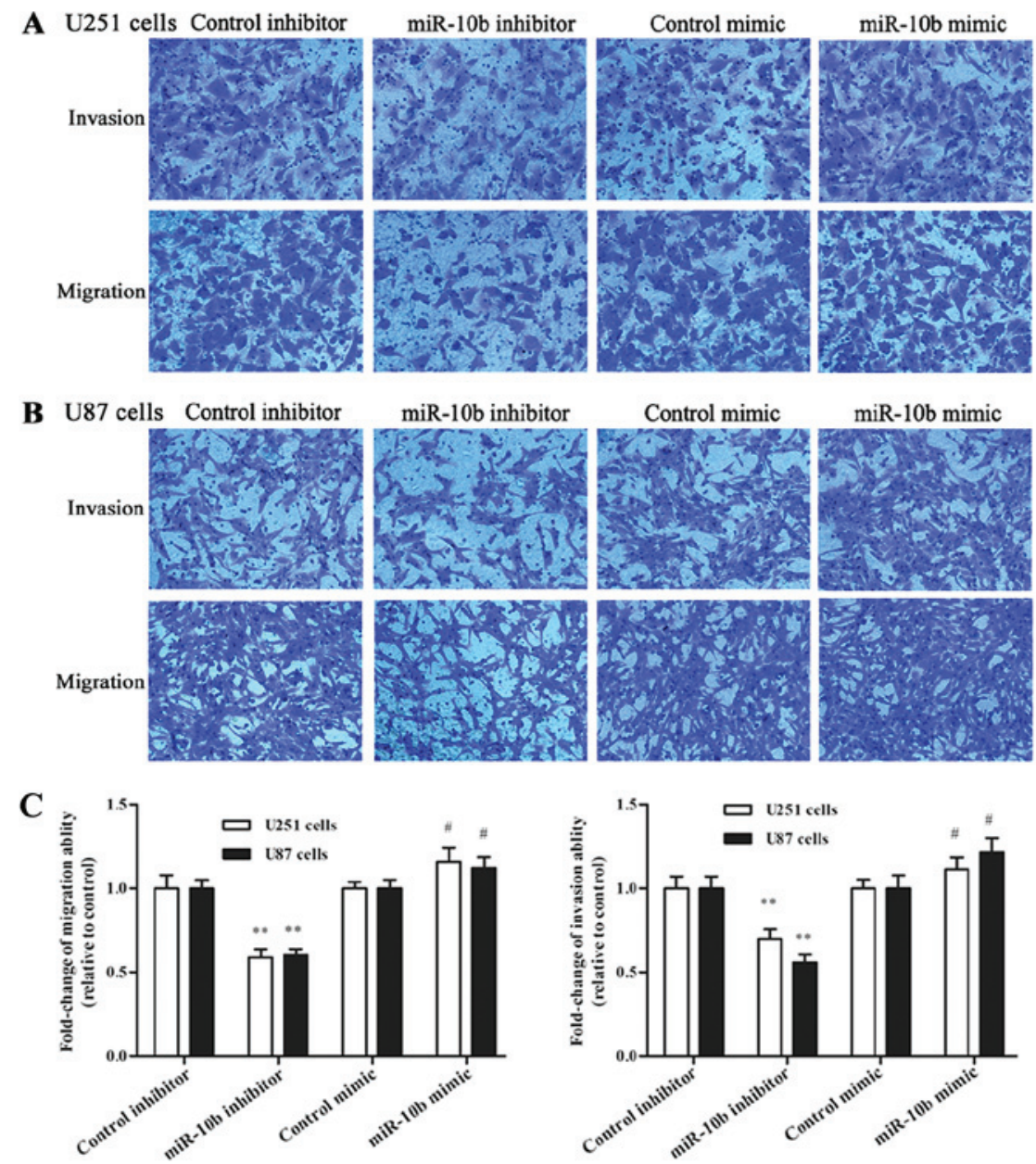

Figure 3. miR-10b-5p regulates glioma cell invasion. (A) U251 and (B) U87 cell invasion and migration Transwell assay following transfected with miR-10b-5p mimic, inhibitor or control (magnification, x200). (C) Graphic representation of the mean migration and invasion percentages. The miR-10b-5p inhibitor group exhibited lower migratory and invasive abilities compared with the control inhibitor group, while the opposite effect was observed in the miR-10b-5p mimic group. ${ }^{* *} \mathrm{P}<0.01$ vs. control inhibitor group; ${ }^{*} \mathrm{P}<0.05$ vs. control mimic group. miR, microRNA. 

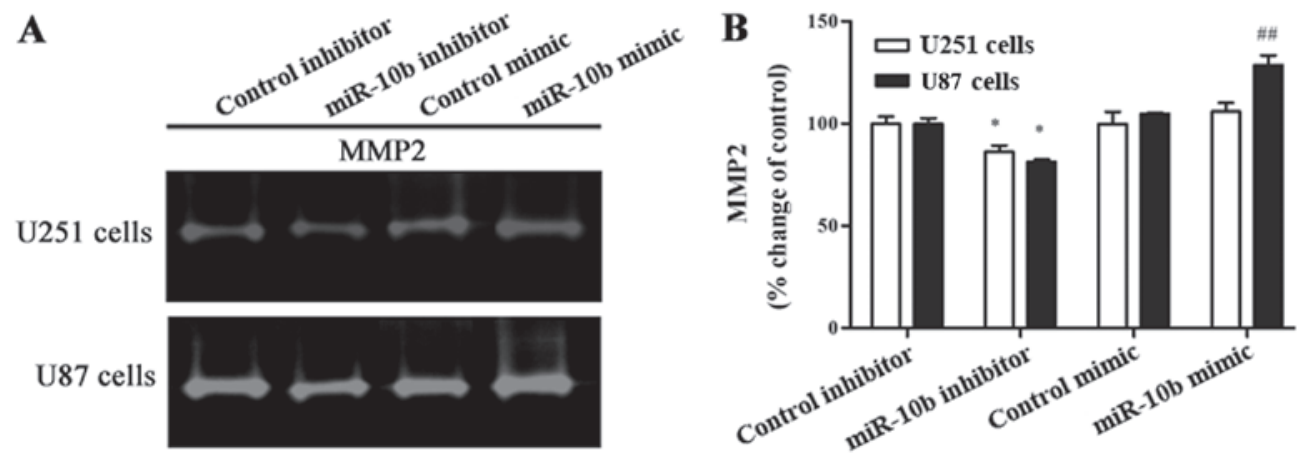

Figure 4. Downregulation of miR-10b-5p inhibits invasion via MMP2 regulation. (A) A gelatin zymography assay was conducted to assess the gelatinase activity changes of MMP2 in U251 and U87 cells. Areas of proteolytic activity appear as clear bands. (B) Quantitative evaluation of MMP2 protein secretions on gelatin zymography. Results were expressed as the percent change of the corresponding control. Data were analyzed by Student's t-test. "P $<0.05$ vs. control inhibitor group; ${ }^{\# \#} \mathrm{P}<0.01$ vs. control mimic group. miR, microRNA; MMP2, matrix metalloproteinase 2.

inhibitor group when compared with the control inhibitor group in glioma cells $(\mathrm{P}<0.05)$. In the miR-10b-5p mimic group, a marked increase in MMP2 secretion was only observed in U87 cells, but not in U251 cells. Collectively, the results indicate that downregulated miR-10b-5p inhibits the invasion of glioma cells by downregulating the expression of MMP-2.

miR-10b-5p modulates HOXB3 and invasion-associated gene expression in glioma cells. A previous study identified HOXB3 as a target gene of miR-10b (14). In the current study, the TargetScan prediction tool was used, and the analysis revealed that HOXB3 contained potential binding sites for miR-10b-5p (Fig. 5A). To identify whether miR-10b-5p can regulate HOXB3 expression in glioma cell lines, the mRNA level of HOXB3 in miR-10b-5p mimic- or inhibitor-transfected glioma cells was first examined. In U251 cells, miR-10b-5p overexpression led to a marked decrease in HOXB3 mRNA level, while miR-10b-5p downregulation significantly increased HOXB3 mRNA (Fig. 5B). Western blotting further revealed that the HOXB3 protein expression level was significantly downregulated following miR-10b-5p mimic transfection in both U251 and U87 cells ( $<<0.05$; Fig. 5C and D). By contrast, the miR-10b-5p inhibitor transfection effectively increased the HOXB3 protein expression level (Fig. 5C and D). These results indicated that the HOXB3 gene may be a target of miR-10b-5p.

It has been reported that $\mathrm{RhoC}$, a member of the Ras homolog gene family, is critical for invasion and metastasis, and is specifically upregulated in glioma (18). In order to investigate whether RhoC is regulated by miR-10b-5p, its protein expression was detected with western blotting. Consistently, the results demonstrated that RhoC was significantly downregulated by miR-10b-5p in both $\mathrm{U} 251$ and $\mathrm{U} 87$ cells $(\mathrm{P}<0.05$; Fig. 5C and D). Furthermore, the protein expression of HMGB1 was evaluated, which has been reported to be involved in the malignant phenotype of glioma cells (19). As shown in Fig. 5C and D, miR-10b-5p inhibition significantly reduced the protein expression of HMGB1, while upregulation of this miRNA markedly increased HMGB1 protein $(\mathrm{P}<0.05)$.

\section{Discussion}

Several microRNAs have recently been identified as either promoters or suppressors of glioma cell invasion and metastasis.
miR-10b is known to be induced by the metastasis-promoting transcription factor Twist (20). The present study data from MTT and cell cycle assays demonstrated that downregulated miR-10b-5p inhibited U251 and U87 cell proliferation, and caused cell cycle arrest. These findings are consistent with those of a previous study by Gabriely et al (6), which reported that miR-10b inhibition led to reduction of glioma cell growth and caused cell cycle arrest at G2 phase in U251 cells, whereas it induced accumulation of U87 cells in the G1 phase. Further wound healing, Transwell and gelatin zymography assays conducted in the present study demonstrated that miR-10b-5p inhibition prevented glioma cell migration and invasion, whereas miR-10b-5p mimic promoted the migration and invasion of both U87 and U251 cells. Previous research revealed that U87 cells lost their invasion potential upon miR-10b inhibition, as well as exhibited reduced MMP2 protein expression, while the invasive ability of U251 cells was enhanced by miR-10b mimic transfection $(21,22)$. Taken together, the current study results combined with previous data suggest an important role for miR-10b-5p in the regulation of glioma cell growth.

HOX genes are known to be master genes encoding a subset of highly conserved transcription factors that serve key roles in the morphogenesis of tissues and organ development in embryos, and their dysregulation is implicated in various types of cancer, including glioma (23). As a member of the HOX gene family, HOXB3 is known as a regulator of gene transcription during development. In fact, HOXB3 has been reported to serve critical roles in cancer initiation and progression, which was confirmed in breast (24), prostate (25), pancreatic (14) and endometrial cancer (13). However, the role of HOXB3 in glioma remains unclear. The findings of the present study indicated a significant association between HOXB3 and miR-10b-5p. HOXB3 expression was upregulated at the mRNA and protein levels in glioma cells with inhibition of miR-10b-5p. These results indicated that HOXB3 may be a downstream target gene of miR-10b-5p in glioma.

In the present study, RhoC expression was regulated by transfection with miR-10b-5p mimic and inhibitor, and its alteration was inversely associated with that of HOXB3 expression. Overexpression of miR-10b-5p upregulated RhoC expression and downregulated HOXB3 expression. Previous correlative studies indicated that miR-10b targeted HOXD10 in glioma 
$\mathbf{A}$

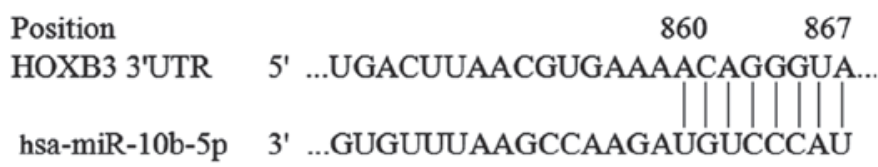

C

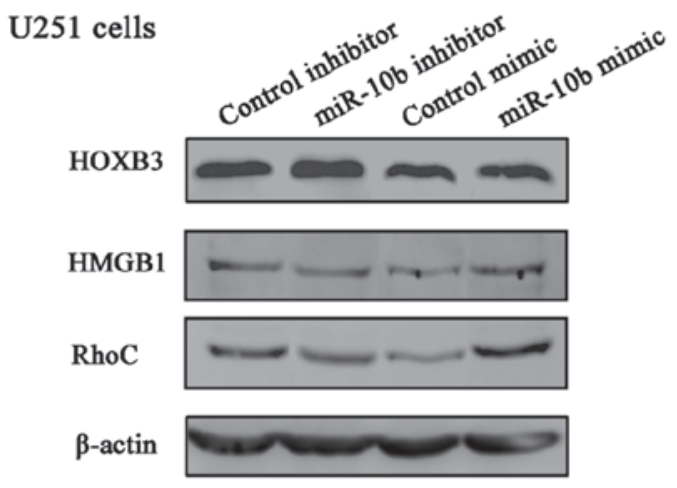

D

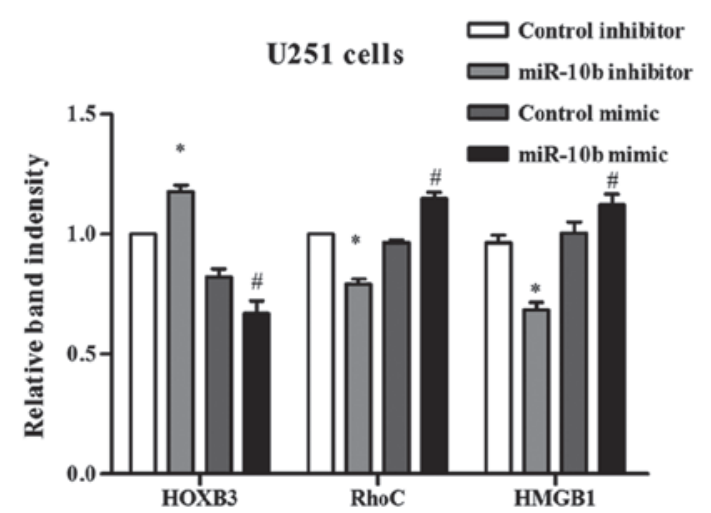

B

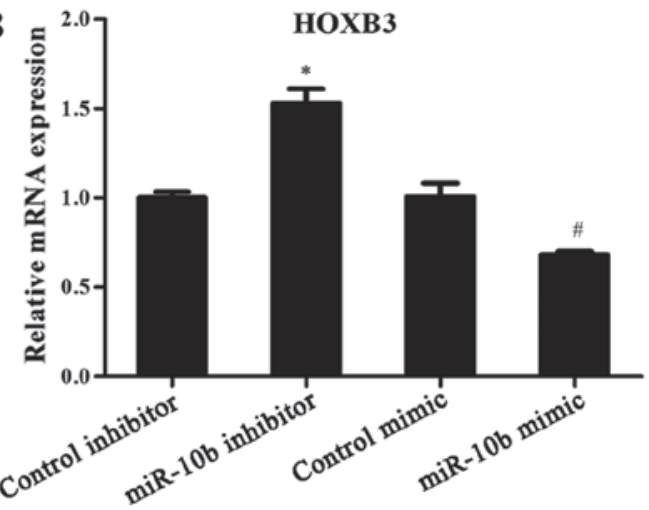

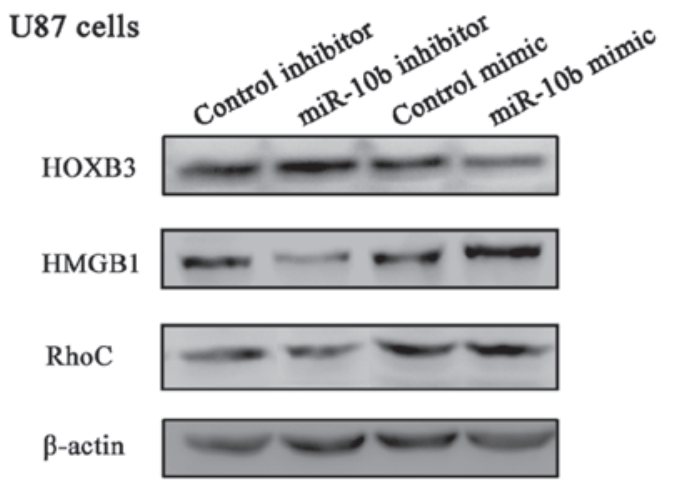

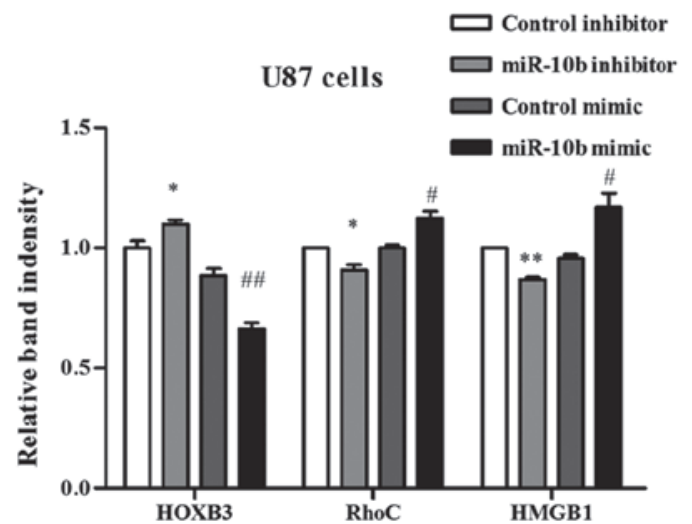

Figure 5. Effect of miR-10b-5p on the expression levels of HOXB3, HMGB1 and RhoC in glioma cells. (A) miR-10b-5p binding sites in the 3'UTR of HOXB3 mRNA, predicted by TargetScan. (B) Reverse transcription-quantitative polymerase chain reaction analysis for HOXB3 in glioma cells transfected with miR-10b-5p mimic, inhibitor or control, indicating that miR-10b-5p regulated the mRNA level of HOXB3. (C) Western blots and (D) quantified results of the expression levels of HOXB3, HMGB1 and RhoC in human glioma cells treated with miR-10b-5p mimic, miR-10b-5p inhibitor or control. The expression of $\beta$-actin served as an endogenous control. miR-10b-5p inhibitor significantly enhanced HOXB3 protein expression, and decreased the downstream RhoC and HMGB1 protein expression levels in U251 and U87 cells. miR-10b-5p mimic exerted the opposite effect. Data were analyzed by Student's t-test. "P<0.05 and ${ }^{* *} \mathrm{P}<0.01$ vs. control inhibitor group; ${ }^{~} \mathrm{P}<0.05$ and ${ }^{\# \#} \mathrm{P}<0.01$ vs. control mimic group. miR, microRNA; 3'UTR, 3'-untranslated region; HOXB3, homeobox B3; RhoC, Ras homolog family member C; HMGB1, high mobility group box 1 .

cells, and HOXD10 was reported to suppress the expression of genes involved in tumor metastasis, including RhoC $(9,22)$. However, it has yet to be determined whether HOXD10 signaling exerts a cross-link effect on HOXB3 regulation in glioma cells, since miR-10b functions as a powerful oncogenic miRNA directly targeting different genes. Future experiments may help elucidate the different miR-10b-associated molecular pathways regulating glioma development.

HMGB1, as a member of the HMGB family, has been found to be overexpressed in various tumors, including human glioma (19). It has been reported that HMGB1 was significantly upregulated in glioma cells, whereas inhibition of
HMGB1 suppressed the proliferation and migration of glioma cells, and promoted apoptosis $(19,26)$. HMGB1 participates in the crosstalk with multiple miRNAs, and promotes metastasis and invasion in glioma. HMGB1 is target gene of miR-218 in glioma cells, which directly suppresses the HMGB1-RAGE pathway (27). miR-129-2 targets HMGB1, thereby suppressing glioma cell growth, migration and invasion, and promoting cell apoptosis (28). In the present study, the regulation of HMGB1 protein expression by miR-10b-5p was similar with that of RhoC expression, with inhibition of miR-10b-5p triggering a significant reduction in the protein expression of HMGB1. It has been reported that HMGB1 induces glioma cell growth 
and migration through its intracellular signaling pathways, including nuclear factor- $\kappa \mathrm{B}$, mitogen-activated protein kinase and extracellular signal-regulated kinase $(29,30)$, suggesting that the mechanism of action of miR-10b may be involved in glioma invasion. Further studies are needed to elucidate the underlying molecular mechanism. To the best of our knowledge, the data reported in the present study are the first to demonstrate an association of the expression level of miR-10b-5p with HOXB3 and HMGB1 in glioma.

A limitation of the present study is that the results illustrated the effect of miR-10b-5p on the invasion ability of glioma cells in vitro. In the future, animal models will also be applied to our study to comprehensively evaluate the effect of miR-10b-5p in the glioma. Furthermore, although the current data demonstrated that there was an inverse correlation between expression of the miR-10b-5p and that of HOXB3 mRNA and protein, it is important to consolidate this finding using dual-luciferase reporter assay, as well as rescue experiments. It is thus recommended that the exact correlation between miR-10b and HOXB3 in the invasion of glioma cells is investigated in future studies.

In conclusion, miR-10b-5p mimic and inhibitor were demonstrated to serve key roles in the regulation of U87 and U251 cell proliferation and invasion capacities in the present study. Transfection with miR-10b-5p inhibitor suppressed glioma cell growth, while miR-10b-5p mimic promoted the migration and invasion of glioma cells. Downregulation of miR-10b-5p increased the expression of HOXB3, resulting in a marked decrease in HMGB1 and RhoC protein levels. These results expanded the list of miR-10b-moderated genes to include HOXB3 in glioma. In addition, the findings provided an insight into the possible mechanism underlying the regulation of glioma by miR-10b-5p, indicating that miR-10b-5p may represent a promising target in glioma treatment.

\section{Acknowledgements}

Not applicable.

\section{Funding}

This study was supported by the Natural Science Foundation of China (grant no. 81302203).

\section{Availability of data and materials}

All data generated or analyzed during the present study are included in this published article.

\section{Authors' contributions}

LL and QR performed the research. QR designed the study. LL and QR analyzed the data. LL wrote the paper. XT provided technical assistance. CL and QX revised the manuscript. All authors have read and approved the final version of this manuscript.

\section{Ethics approval and consent to participate}

Not applicable.

\section{Patient consent for publication}

Not applicable.

\section{Competing interests}

The authors declare that they have no competing interests.

\section{References}

1. Brandes AA, Tosoni A, Franceschi E, Sotti G, Frezza G, Amistà P, Morandi L, Spagnolli F and Ermani M: Recurrence pattern after temozolomide concomitant with and adjuvant to radiotherapy in newly diagnosed patients with glioblastoma: Correlation with MGMT promoter methylation status. J Clin Oncol 27: 1275-1279, 2009.

2. Ru Q, Tian X, Wu YX, Wu RH, Pi MS and Li CY: Voltage-gated and ATP-sensitive $\mathrm{K}+$ channels are associated with cell proliferation and tumorigenesis of human glioma. Oncol Rep 31: 842-848, 2014.

3. Mathupala SP, Mittal S, Guthikonda M and Sloan AE: MicroRNA and brain tumors: A cause and a cure? DNA Cell Biol 26: 301-310, 2007.

4. Ma L, Teruya-Feldstein J and Weinberg RA: Tumour invasion and metastasis initiated by microRNA-10b in breast cancer. Nature 449: 682-688, 2007.

5. Ciafrè SA, Galardi S, Mangiola A, Ferracin M, Liu CG Sabatino G, Negrini M, Maira G, Croce CM and Farace MG: Extensive modulation of a set of microRNAs in primary glioblastoma. Biochem Biophys Res Commun 334: 1351-1358, 2005.

6. Gabriely G, Yi M, Narayan RS, Niers JM, Wurdinger T, Imitola J, Ligon KL, Kesari S, Esau C, Stephens RM, et al: Human glioma growth is controlled by microRNA-10b. Cancer Res 71: 3563-3572, 2011.

7. Ji Y, Wei Y, Wang J, Gong K, Zhang Y and Zuo H: Correlation of microRNA-10b upregulation and poor prognosis in human gliomas. Tumour Biol 36: 6249-6254, 2015.

8. Guessous F, Alvarado-Velez M, Marcinkiewicz L, Zhang Y, Kim J, Heister S, Kefas B, Godlewski J, Schiff D, Purow B and Abounader R: Oncogenic effects of miR-10b in glioblastoma stem cells. J Neurooncol 112: 153-163, 2013.

9. Sasayama T, Nishihara M, Kondoh T, Hosoda K and Kohmura E: MicroRNA-10b is overexpressed in malignant glioma and associated with tumor invasive factors, $\mathrm{uPR}$ and $\mathrm{RhoC}$. Int J Cancer 125: 1407-1413, 2009.

10. Lin J, Teo S, Lam DH, Jeyaseelan K and Wang S: MicroRNA-10b pleiotropically regulates invasion, angiogenicity and apoptosis of tumor cells resembling mesenchymal subtype of glioblastoma multiforme. Cell Death Dis 3: e398, 2012.

11. Ru Q, Li WL, Xiong Q, Chen L, Tian X and Li CY: Voltage-gated potassium channel blocker 4-aminopyridine induces glioma cell apoptosis by reducing expression of microRNA-10b-5p. Mol Biol Cell 29: 1125-1136, 2018.

12. Weiss FU, Marques IJ, Woltering JM, Vlecken DH, Aghdassi A, Partecke LI, Heidecke CD, Lerch MM and Bagowski CP: Retinoic acid receptor antagonists inhibit miR-10a expression and block metastatic behavior of pancreatic cancer. Gastroenterology 137: 2136-2145, 2009

13. Chen H, Fan Y, Xu W, Chen J, Xu C, Wei X, Fang D and Feng Y: miR-10b inhibits apoptosis and promotes proliferation and invasion of endometrial cancer cells via targeting HOXB3. Cancer Biother Radiopharm 31: 225-231, 2016.

14. Yang D, Yan R, Zhang X, Zhu Z, Wang C, Liang C and Zhang X: Deregulation of MicroRNA-375 inhibits cancer proliferation migration and chemosensitivity in pancreatic cancer through the association of HOXB3. Am J Transl Res 8: 1551-1559, 2016.

15. Hussain R, Umer HM, Björkqvist M and Roomans GM: ENaC, iNOS, mucins, and wound healing in cystic fibrosis airway epithelial and submucosal cells. Cell Biol Int Rep 21: 25-38, 2014.

16. Livak KJ and Schmittgen TD: Analysis of relative gene expression data using real-time quantitative PCR and the 2(-Delta Delta C(T)) method. Methods 25: 402-408, 2001.

17. Mayes DA, Hu Y, Teng Y, Siegel E, Wu X, Panda K, Tan F, Yung WK and Zhou YH: PAX6 suppresses the invasiveness of glioblastoma cells and the expression of the matrix metalloproteinase-2 gene. Cancer Res 66: 9809-9817, 2006. 
18. Huang H, Okamoto Y, Yokoo H, Heppner FL, Vital A, Fevre-Montange M, Jouvet A, Yonekawa Y, Lazaridis EN, Kleihues $\mathrm{P}$ and Ohgaki $\mathrm{H}$ : Gene expression profiling and subgroup identification of oligodendrogliomas. Oncogene 23: 6012-6022, 2004.

19. Angelopoulou E,Piperi C, Adamopoulos $C$ and Papavassiliou AG: Pivotal role of high-mobility group box 1 (HMGB1) signaling pathways in glioma development and progression. J Mol Med (Berl) 94: 867-874, 2016.

20. Gabriely G, Teplyuk NM and Krichevsky AM: Context effect: microRNA-10b in cancer cell proliferation, spread and death. Autophagy 7: 1384-1386, 2011.

21. Sun L, Yan W, Wang Y, Sun G, Luo H, Zhang J, Wang X, You Y, Yang Z and Liu N: MicroRNA-10b induces glioma cell invasion by modulating MMP-14 and UPAR expression via HOXD10 Brain Res 1389: 9-18, 2011.

22. Dong CG, Wu WK, Feng SY, Wang XJ, Shao JF and Qiao J: Co-inhibition of microRNA-10b and microRNA-21 exerts synergistic inhibition on the proliferation and invasion of human glioma cells. Int J Oncol 41: 1005-1012, 2012.

23. Shah N and Sukumar S: The Hox genes and their roles in oncogenesis. Nat Rev Cancer 10: 361-371, 2010.

24. Fu H, Fu L, Xie C, Zuo WS, Liu YS, Zheng MZ and Yu JM: miR-375 inhibits cancer stem cell phenotype and tamoxifen resistance by degrading HOXB3 in human ER-positive breast cancer. Oncol Rep 37: 1093-1099, 2017.
25. Chen J, Zhu S, Jiang N, Shang Z, Quan C and Niu Y: HoxB3 promotes prostate cancer cell progression by transactivating CDCA3. Cancer Lett 330: 217-224, 2013.

26. Zhang J, Liu C and Hou R: Knockdown of HMGB1 improves apoptosis and suppresses proliferation and invasion of glioma cells. Chin J Cancer Res 26: 658-668, 2014.

27. Gu J, Xu R, Li Y, Zhang J and Wang S: MicroRNA-218 modulates activities of glioma cells by targeting HMGB1. Am J Transl Res 8: 3780-3790, 2016

28. Yang Y, Huang JQ, Zhang X and Shen LF: MiR-129-2 functions as a tumor suppressor in glioma cells by targeting HMGB1 and is down-regulated by DNA methylation. Mol Cell Biochem 404: 229-239, 2015.

29. Fiuza C, Bustin M, Talwar S, Tropea M, Gerstenberger E, Shelhamer JH and Suffredini AF: Inflammation-promoting activity of HMGB1 on human microvascular endothelial cells. Blood 101: 2652-2660, 2003.

30. Treutiger CJ, Mullins GE, Johansson AS, Rouhiainen A, Rauvala HM, Erlandsson-Harris H, Andersson U, Yang H, Tracey KJ, Andersson J and Palmblad JE: High mobility group 1 B-box mediates activation of human endothelium. J Intern Med 254: 375-385, 2003.

This work is licensed under a Creative Commons Attribution-NonCommercial-NoDerivatives 4.0 International (CC BY-NC-ND 4.0) License. 